\title{
空气污染各组分对甲烷超声速燃烧性能的影响
}

\author{
侯凌云 ${ }^{1, *}$ 杨 缙 ${ }^{1}$ 马雪松 ${ }^{2}$ 刘 巍 ${ }^{2}$ \\ ('清华大学航天航空学院, 北京 $100084 ; 2$ 北京动力机械研究所, 北京 100074)
}

\begin{abstract}
摘要: 在与甲烷详细化学反应机理对比验证基础上, 采用 18 组分 24 步简化反应机理模拟甲烷超声速燃烧过 程, 从化学动力学和热力学角度用数值方法研究了乙醇燃烧加热空气中的七种主要污染组分 $\left(\mathrm{H}_{2} \mathrm{O}, \mathrm{CO}_{2}, \mathrm{O}\right.$, $\mathrm{OH}, \mathrm{CO}, \mathrm{H}, \mathrm{H}_{2}$ ) 对甲烷超声速燃烧性能的影响. 分析结果表明: 在一定条件下, 进口空气中污染组分 $\mathrm{H}_{2} \mathrm{O}$ 的增加 造成平均比热容增加, 总温降低, 并作为第三体抑制甲烷的燃烧过程, 使超燃室的性能下降; $\mathrm{CO}_{2}$ 因大分子量特 性使燃气平均分子量增大, 降低超燃室做功能力, $\mathrm{H}_{2} \mathrm{O}$ 和 $\mathrm{CO}_{2}$ 两组分对甲烷超燃性能都起消极作用; 污染组分 自由基 $\mathrm{H} 、 \mathrm{O} 、 \mathrm{OH}$ 和燃烧中间产物 $\mathrm{CO} 、 \mathrm{H}_{2}$ 使燃烧室燃烧效率上升, 对甲烷超燃性能起积极作用.
\end{abstract}

关键词：超声速燃烧；化学反应机理；污染空气；组分 中图分类号: 0643

\section{Effects of Species in Vitiation Air on Methane-Fueled Supersonic Combustion}

\author{
HOU Ling-Yun ${ }^{1, *} \quad$ YANG Jin ${ }^{1} \quad$ MAXue-Song ${ }^{2} \quad$ LIU Wei $^{2}$ \\ ('School of Aerospace, Tsinghua University, Beijing 100084, P. R. China; \\ ${ }^{2}$ Beijing Power Machinery Research Institute, Beijing 100074, P. R. China)
}

\begin{abstract}
Based on a detailed chemical reaction mechanism, a reduced reaction mechanism with 18 species and 24 steps was used to simulate the supersonic combustion of methane. Heated air calculations showed that seven main vitiated species, i.e., $\mathrm{H}_{2} \mathrm{O}, \mathrm{CO}_{2}, \mathrm{O}, \mathrm{OH}, \mathrm{CO}, \mathrm{H}$, and $\mathrm{H}_{2}$, were present in ethanolfueled heated air. We analyzed the effects of these species on methane-fueled supersonic combustion using chemical kinetics and thermodynamics. $\mathrm{H}_{2} \mathrm{O}$ inhibits the combustion process, decreases the combustion efficiency, and decreases the specific thrust. The relatively large molecular weight of $\mathrm{CO}_{2}$ contributes to an increase in the mean molecular weight of the fuel gas, which is a negative factor in the mechanism of specific thrust. Free radicals $\mathrm{O}, \mathrm{OH}, \mathrm{H}$ can effectively promote the combustion process and thus increase the combustion efficiency. Intermediate products $\mathrm{CO}$ and $\mathrm{H}_{2}$ increase the combustion efficiency, and this is a function of the additional fuel.
\end{abstract}

Key Words: Supersonic combustion; Chemical reaction mechanism; Vitiation air; Species

超燃冲压发动机地面实验中要再现飞行时的高 速高焓气流特性, 必须对进口气体进行预加热以使 总焓达到高飞行马赫数时气流总焓. 目前, 主要采用 电弧加热和燃烧加热两种方式对空气进行加热, 以 模拟高空高焓气流条件. 在工程应用中, 常使用比电
加热更经济的燃烧加热方式来模拟高空高速、高焓 进口气流. 然而, 燃烧加热空气与实际空气组分组 成、比热、分子量等的差异会使地面实验结果不能等 同于飞行实验结果. 于是, 如何使用污染空气来模拟 和复现超声速燃烧飞行状态下的实际性能, 研究燃

Received: May 10, 2010; Revised: August 13, 2010; Published on Web: October 26, 2010.

"Corresponding author. Email: lyhou@tsinghua.edu.cn; Tel: +86-10-62772157.

The project was supported by the National Natural Science Foundation of China (50306011).

国家自然科学基金(50306011)资助项目

(C) Editorial office of Acta Physico-Chimica Sinica 
烧加热污染空气对超声速燃烧的影响就成为燃烧实 验技术需要解决的重要课题.

国外加热空气污染物影响超音速燃烧的研究 始于 1960-1970年, 人们从理论、实验和数值模拟 来研究燃烧加热后的污染空气对超音速燃烧过程 的影响 ${ }^{[1-3]}$. 但其发表的文献大多是针对氢超燃过程 影响的分析. 国内也在近几年展开了相关研究 ${ }^{[4-6]}$, 但大多侧重在对最终产物影响的计算和实验. 本文 针对乙醇燃烧加热空气中的主要污染物, 包括最终 产物 $\mathrm{H}_{2} \mathrm{O} 、 \mathrm{CO}_{2}$, 自由基 $\mathrm{O} 、 \mathrm{OH} 、 \mathrm{H}$, 中间产物 $\mathrm{CO} 、 \mathrm{H}_{2}$, 对甲烷超声速燃烧性能影响展开数值模拟.

\section{1 计算模型与方法}

\section{1 燃烧室模型}

数值计算所使用的燃烧室模型如图 1 所示, 为 具有中心支板和两对凹槽的突扩型双模态超燃冲压 发动机燃烧室 ${ }^{[7]}$. 此模型的支板燃料注入系统能够 使燃料在高速气流中均匀分布, 并在很短的时间内 完成混合, 凹槽火焰稳定器能对点火和稳定燃烧起 积极作用. 燃料从燃烧室壁面距凹槽 $15 \mathrm{~mm}$ 处径向 垂直喷入, 以及支板尾缘顺向喷入主流. 由于结构的 对称性, 取其一半进行计算. 采用结构化网格, 对支 板、凹槽及壁面进行了局部加密, 网格数为 15265 .

\section{2 控制方程与模型}

采用包含多种组分带化学反应的雷诺平均、守 恒型 Navier-Stokes 方程作为气体湍流流动、燃烧控 制方程:

$$
\frac{\partial \boldsymbol{U}}{\partial t}+\frac{\partial\left(\boldsymbol{F}-\boldsymbol{F}_{v}\right)}{\partial x}+\frac{\partial\left(\boldsymbol{G}-\boldsymbol{G}_{y}\right)}{\partial y}=\boldsymbol{H}
$$

式中 $\boldsymbol{U}$ 为守恒变量向量, $\boldsymbol{F 、} 、 \boldsymbol{G}$ 为对流项向量, $\boldsymbol{F}_{v} 、 \boldsymbol{G}_{v}$

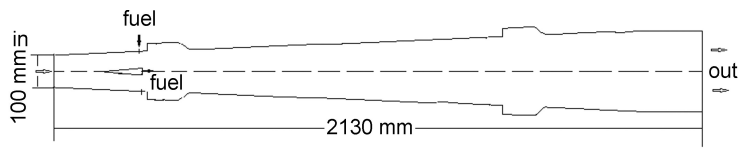

图 1 超燃室结构简图 ${ }^{171}$

Fig.1 Schematic structure of supersonic combustor ${ }^{[7]}$ 为粘性项向量, $\boldsymbol{H}$ 为源项向量. 其中:

$$
\begin{aligned}
& \boldsymbol{U}=\left(\rho, \rho u, \rho v, \rho E, \rho Y_{1}, \cdots, \rho Y_{i}\right)^{T} \\
& \boldsymbol{F}=\left(\rho u, \rho u^{2}+p, \rho u v,(\rho E+p) u, \rho u Y_{1}, \cdots, \rho u Y_{i}\right)^{T} \\
& \boldsymbol{G}=\left(\rho v, \rho u v, \rho v^{2}+p,(\rho E+p) v, \rho v Y_{1}, \cdots, \rho v Y_{i}\right)^{T} \\
& \boldsymbol{F}_{v}=\left(0, \tau_{x x}, \tau_{x y}, u \tau_{x x}+v \tau_{x y}+q_{x e}, \tau_{x k}, \tau_{x E}, q_{x 1}, \cdots, q_{x i}\right)^{T} \\
& \boldsymbol{G}_{v}=\left(0, \tau_{y x}, \tau_{y y}, u \tau_{y x}+v \tau_{y y}+q_{y e}, \tau_{y k}, \tau_{y E}, q_{y 1}, \cdots, q_{y i}\right)^{T} \\
& \boldsymbol{H}=\left(0,0,0, S_{d, h}, \omega_{1}, \cdots, \omega_{i}\right)^{T}
\end{aligned}
$$

其中 $\rho$ 为密度, $u 、 v$ 为轴向和径向速度分量, $E 、 p$ 为内 能和压力, $Y_{i}$ 为 $i$ 组分的质量分数, $\tau_{i j}$ 和 $q_{i}$ 为粘性应力 分量和反应吸放热量, $S_{d, h}$ 为化学反应作用源项, $\omega_{i}$ 为组分 $i$ 的质量生成率. 以 SST(shear-stress transport) $k-\omega$ 湍流模型、空间反应浴耗散概念燃烧模型封闭 雷诺平均方程, 对超声速流和化学反应进行耦合计 算.

\section{3 化学反应动力学模型}

本文参考 Peters 等 ${ }^{[8]}$ 的甲烷 14 组分 18 反应模型 和董刚等 ${ }^{[9]}$ 的甲烷 $Y$ 简化机理模型, 在考虑氮氧化 物生成的条件下, 构造而得甲烷 18 组分 24 反应简化 反应机理模型. 如下表 1 所示.

此甲烷模型中 $\mathrm{R} 1-\mathrm{R} 2$ 为链引发; $\mathrm{R} 3-\mathrm{R} 8$ 为链分 支; R9-R12 为链传递; R13-R19 为链终止 ${ }^{[9]}$, 其余为

\begin{tabular}{|c|c|c|c|c|c|c|c|c|c|}
\hline No. & Reaction & $A$ & $\beta$ & $E$ & No. & Reaction & $A$ & $\beta$ & $E$ \\
\hline $\mathrm{R} 1$ & $\mathrm{H}+\mathrm{CH}_{4} \rightleftharpoons \mathrm{CH}_{3}+\mathrm{H}_{2}$ & $6.60 \times 10^{8}$ & 1.62 & 45528 & R13 & $\mathrm{H}+\mathrm{O}_{2}+\mathrm{M} \rightleftharpoons \mathrm{HO}_{2}+\mathrm{M}$ & $2.80 \times 10^{18}$ & -0.86 & 0 \\
\hline $\mathrm{R} 2$ & $\mathrm{OH}+\mathrm{CH}_{4} \rightleftharpoons \mathrm{CH}_{3}+\mathrm{H}_{2} \mathrm{O}$ & $1.00 \times 10^{8}$ & 1.60 & 13104 & R14 & $\mathrm{H}+\mathrm{OH}+\mathrm{M} \rightleftharpoons \mathrm{H}_{2} \mathrm{O}+\mathrm{M}$ & $2.20 \times 10^{22}$ & -2.00 & 0 \\
\hline R3 & $\mathrm{O}+\mathrm{CH}_{3} \rightleftharpoons \mathrm{H}+\mathrm{CH}_{2} \mathrm{O}$ & $5.06 \times 10^{13}$ & 0.00 & 0 & R15 & $\mathrm{H}+\mathrm{HO}_{2} \rightleftharpoons \mathrm{O}_{2}+\mathrm{H}_{2}$ & $4.48 \times 10^{13}$ & 0.00 & 4486 \\
\hline R4 & $\mathrm{H}+\mathrm{CH}_{2} \mathrm{O} \rightleftharpoons \mathrm{HCO}+\mathrm{H}_{2}$ & $5.74 \times 10^{7}$ & 1.90 & 11516 & R16 & $\mathrm{H}+\mathrm{HO}_{2} \rightleftharpoons 2 \mathrm{OH}$ & $0.84 \times 10^{14}$ & 0.00 & 2667 \\
\hline R5 & $\mathrm{OH}+\mathrm{CH}_{2} \mathrm{O} \rightleftharpoons \mathrm{HCO}+\mathrm{H}_{2} \mathrm{O}$ & $3.43 \times 10^{9}$ & 1.18 & -1877 & R17 & $\mathrm{OH}+\mathrm{HO}_{2} \rightleftharpoons \mathrm{O}_{2}+\mathrm{H}_{2} \mathrm{O}$ & $1.45 \times 10^{13}$ & 0.00 & -2100 \\
\hline R6 & $\mathrm{H}+\mathrm{HCO} \rightleftharpoons \mathrm{H}_{2}+\mathrm{CO}$ & $7.34 \times 10^{13}$ & 0.00 & 0 & R18 & $\mathrm{H}+\mathrm{CH}_{3}+\mathrm{M} \rightleftharpoons \mathrm{CH}_{4}+\mathrm{M}$ & $13.9 \times 10^{15}$ & -0.53 & 2251 \\
\hline R7 & $\mathrm{HCO}+\mathrm{M} \rightleftharpoons \mathrm{H}+\mathrm{CO}+\mathrm{M}$ & $1.87 \times 10^{17}$ & -1.00 & 71400 & R19 & $\mathrm{H}+\mathrm{H}+\mathrm{M} \rightleftharpoons \mathrm{H}_{2}+\mathrm{M}$ & $1.80 \times 10^{18}$ & -1.00 & 0 \\
\hline $\mathrm{R} 8$ & $\mathrm{OH}+\mathrm{CO} \rightleftharpoons \mathrm{H}+\mathrm{CO}_{2}$ & $4.76 \times 10^{7}$ & 1.22 & 294 & $\mathrm{R} 20$ & $\mathrm{~N}+\mathrm{NO} \rightleftharpoons \mathrm{N}_{2}+\mathrm{O}$ & $2.70 \times 10^{13}$ & 0.00 & 1491 \\
\hline R9 & $\mathrm{H}+\mathrm{O}_{2} \rightleftharpoons \mathrm{O}+\mathrm{OH}$ & $2.65 \times 10^{16}$ & -0.67 & 71572 & $\mathrm{R} 21$ & $\mathrm{~N}+\mathrm{O}_{2} \rightleftharpoons \mathrm{NO}+\mathrm{O}$ & $9.00 \times 10^{9}$ & 1.00 & 27300 \\
\hline $\mathrm{R} 10$ & $\mathrm{O}+\mathrm{H}_{2} \rightleftharpoons \mathrm{H}+\mathrm{OH}$ & $3.87 \times 10^{4}$ & 2.70 & 26292 & R22 & $\mathrm{CH}_{3}+\mathrm{NO} \rightleftharpoons \mathrm{HCN}+\mathrm{H}_{2} \mathrm{O}$ & $9.60 \times 10^{13}$ & 0.00 & 120960 \\
\hline R11 & $\mathrm{OH}+\mathrm{H}_{2} \rightleftharpoons \mathrm{H}+\mathrm{H}_{2} \mathrm{O}$ & $2.16 \times 10^{8}$ & 1.51 & 14406 & $\mathrm{R} 23$ & $\mathrm{HO}_{2}+\mathrm{NO} \rightleftharpoons \mathrm{NO}_{2}+\mathrm{OH}$ & $2.11 \times 10^{12}$ & 0.00 & -480 \\
\hline R12 & $\mathrm{OH}+\mathrm{OH} \rightleftharpoons \mathrm{H}_{2} \mathrm{O}+\mathrm{O}$ & $6.00 \times 10^{8}$ & 1.30 & 0 & R24 & $\mathrm{NO}+\mathrm{O}+\mathrm{M} \rightleftharpoons \mathrm{NO}_{2}+\mathrm{M}$ & $1.06 \times 10^{20}$ & -1.41 & 0 \\
\hline
\end{tabular}
氮氧化物生成反应. 利用 Chemkin 4.0 中的一维对冲 扩散火焰模型对甲烷 18 组分 24 步简化反应机理与 甲烷 53 组分 325 步详细反应机理(主要取自 GRI$\left.\mathrm{MECH} 2.11^{[10]}\right)$ 进行对比、验证. 两者的温度分布和主

表 1 甲烷燃烧化学反应动力学模型

Table 1 Chemical reaction mechanism of methane combustion

$A$ : pre-exponential factor $\left(\mathrm{mol} \cdot \mathrm{s} \cdot \mathrm{cm}^{3}\right) ; E$ : activation energy for the reaction $\left(\mathrm{J} \cdot \mathrm{mol}^{-1}\right) ; \beta$ : temperature exponent 


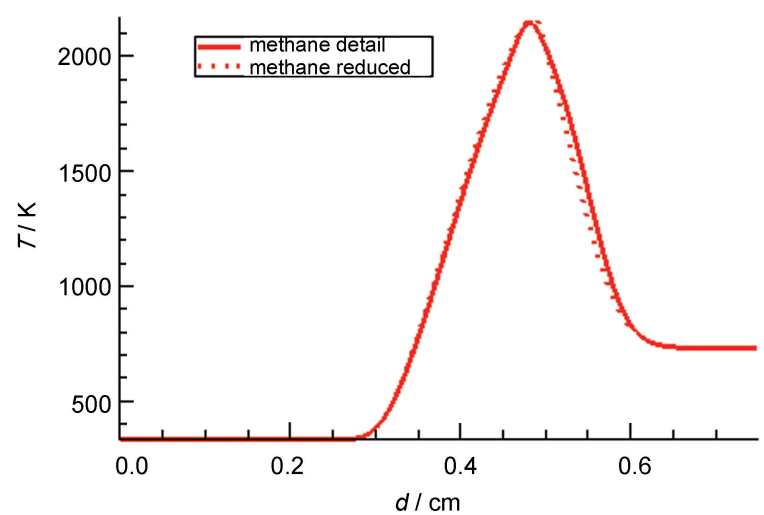

图 2 温度分布

Fig.2 Temperature distributions

$d$ is the distance from the fuel injector to the oxidizer injector.

要组分分布比较见图2、图3 和图4.

图 2、图 3、图 4中横坐标 $d$ 代表从燃料喷口到氧 化剂喷口的距离, 图 3 和图 4 左边的纵坐标代表 $\mathrm{O}_{2}$ 和 $\mathrm{CH}_{4}$ 的摩尔分数, 右边的纵坐标 $x$ 代表 $\mathrm{H}_{2} 、 \mathrm{H}_{2} \mathrm{O}$ 、 $\mathrm{HO}_{2} 、 \mathrm{CH}_{3} 、 \mathrm{CO}$ 和 $\mathrm{CO}_{2}$ 的摩尔分数. 可以看出, 两者的 温度分布和主要组分分布基本上是一致的, 说明本 文构造的甲烷 18 组分 24 步简化反应机理与 53 组分 水岭 325 步详细反应机理较好吻合, 能够反映出各 成分的变化规律.

\section{4 边界条件与进口空气组分}

为获得超燃室入口条件, 首先需对乙醇加热燃 烧器展开其研究. 对于乙醇加热器而言, 其入口条件 为: 空气质量流量为 $0.067 \mathrm{~kg} \cdot \mathrm{s}^{-1}$, 氧气的质量流量 为 $0.016 \mathrm{~kg} \cdot \mathrm{s}^{-1}$, 乙醇的质量流量为 $0.0077 \mathrm{~kg} \cdot \mathrm{s}^{-1}$, 燃 烧完成后, 保证出口燃气中氧气的摩尔分数仍为 $21 \%$, 燃气总温为 $1644 \mathrm{~K}$. 采用 Chemkin 和 Lawrence 实验室的 68 组分 388 步反应乙醇燃烧机理 ${ }^{[1]}$ 对乙醇 燃烧加热器燃烧过程进行模拟, 得到加热器出口(超 燃室入口)主要组分含量, 出口燃气中除了 $\mathrm{N}_{2} 、 \mathrm{O}_{2}$ 外,

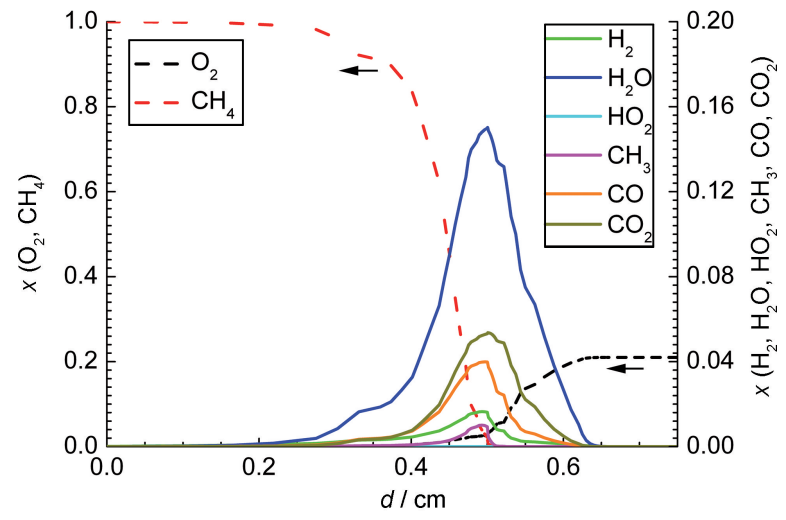

图 3 简化机理的主要组分

Fig.3 Main species of reduced mechanism

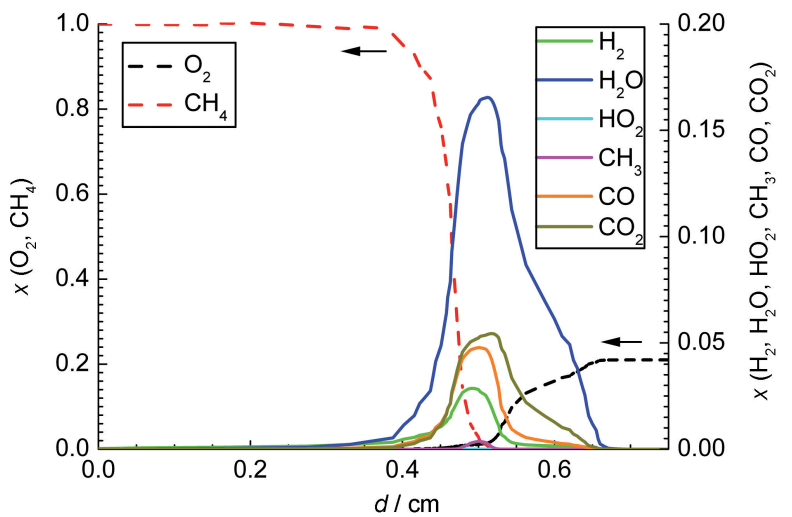

图 4 详细机理的主要组分

Fig.4 Main species of detailed mechanism

含量较多的 7 种组分分别是 $\mathrm{H}_{2} \mathrm{O} 、 \mathrm{CO}_{2} 、 \mathrm{O} 、 \mathrm{OH} 、 \mathrm{CO}$ 、 $\mathrm{H} 、 \mathrm{H}_{2}$. 其中 $\mathrm{H}_{2} \mathrm{O}$ 和 $\mathrm{CO}_{2}$ 含量占的比重非常大, 摩尔分 数分别在 $7 \%$ 和 $10 \%$ 左右; $\mathrm{O} 、 \mathrm{OH} 、 \mathrm{CO}$ 含量在同一个 数量级 $10^{-3} ; \mathrm{H} 、 \mathrm{H}_{2}$ 的含量在同一个数量级 $10^{-4}$. 其余 组分如 $\mathrm{C}_{2} \mathrm{H}_{4} 、 \mathrm{C}_{2} \mathrm{H}_{2} 、 \mathrm{CH}_{4} 、 \mathrm{CH}_{3} 、 \mathrm{CH}_{2} \mathrm{O} 、 \mathrm{HO}_{2}$ 等, 摩尔分 数数量级在 $10^{-6}$ 以下, 这些组分不会对超声速燃烧 造成明显影响, 因此主要考虑 7 种组分即最终产物 $\left(\mathrm{H}_{2} \mathrm{O} 、 \mathrm{CO}_{2}\right)$ 、自由基 $(\mathrm{O} 、 \mathrm{OH} 、 \mathrm{H})$ 和中间产物 $\left(\mathrm{CO} 、 \mathrm{H}_{2}\right)$.

空气入口采用压力入口, 总压强 $732152.8 \mathrm{~Pa}$, 静压强 $42852.9 \mathrm{~Pa}$, 总温度 $1644.15 \mathrm{~K}$. 燃料入口为压 力入口, 以声速喷入常温 $(300 \mathrm{~K})$ 甲烷, 其流量按燃 烧当量比 0.723 给定, 第一个凹槽前沿与支板尾缘燃 料喷注比为 6:1. 出口为压力出口, 背压为大气环境 压力 $101325 \mathrm{~Pa}$, 总温 $300 \mathrm{~K}$, 壁面为无滑移绝热壁 面. 表 2-4 为分别改变 $\mathrm{H}_{2} \mathrm{O} 、 \mathrm{CO}_{2} 、 \mathrm{O} 、 \mathrm{OH} 、 \mathrm{CO} 、 \mathrm{H} 、 \mathrm{H}_{2}$ 的摩尔分数, 与 $\mathrm{O}_{2} 、 \mathrm{H}_{2} \mathrm{O}$ 和 $\mathrm{N}_{2}$ (剩余含量计入 $\mathrm{N}_{2}$ 组分 中)构成不同进口气流工况. 其中每组工况中带“\#” 号的为基准工况 (电加热空气工况), 带“*”号的工况 中的单个组分含量值为乙醇燃烧加热污染空气中该 组分的含量. 当七种组分中每种组分摩尔含量改变, 而其余成分不变时 (指 $\mathrm{O}_{2}$ 和 $\mathrm{H}_{2} \mathrm{O}$ ), 研究各组分对超 燃性能的影响.

\section{5 性能参数定义 ${ }^{[1,12]}$}

(1) 燃烧效率

本文选择基于总温变化来计算燃烧效率. 定义 如下:

$$
\eta_{c(\Delta T)}=\frac{T_{\mathrm{t}}-T_{0}}{T_{0, \text { ideal }}-T_{0}}
$$

其中质量平均总温 $T_{t}$ 定义为

$T_{\mathrm{t}}=\int \rho u T_{\mathrm{t}, A} \mathrm{~d} A / \int \rho u \mathrm{~d} A$

绝对火焰温度 $T_{0, \text { ideal }}$ 为 


$$
-\Delta H_{\mathrm{b}}^{0}=\Delta H_{\mathrm{pr}}=\int_{T_{0}}^{T_{0, \text { ideal }}} \sum_{j, \mathrm{pr}} n_{j} C_{p, m, j} \mathrm{~d} T
$$

其中, $T_{0}$ 为入口的质量平均总温, $\Delta H_{\mathrm{b}}^{0}$ 为定压标准燃 烧热; $n_{j} 、 C_{p, m, j}$ 分别为燃烧产物中第 $j$ 组元的摩尔数及 摩尔定压热容, pr 为生成物.

(2) 燃烧室参考比推力

推力由压差和动量差两部分组成, 定义如下:

$$
F=\left(\int p \mathrm{~d} A+\int \rho u^{2} \mathrm{~d} A\right)_{\text {inlet }}^{\text {exit }}
$$

比推力 $f$ 定义为超燃室参考推力与燃气质量流量之 比, 即 $f=F / \dot{m}$. 其中 $F$ 为超燃室参考推力, $\dot{m}$ 为燃 气质量流量.

(3) 出口截面燃气平均定压比热容和平均分子量:

$$
\begin{gathered}
C_{p, \text { av }}=\sum_{i} \frac{\int \rho u n_{i} C_{p, i} \mathrm{~d} A}{\int \rho u \mathrm{~d} A} \\
M_{\mathrm{av}}=\sum_{i} \frac{\int \rho u n_{i} M_{i} \mathrm{~d} A}{\int \rho u \mathrm{~d} A}
\end{gathered}
$$

其中, $n_{i}$ 为第 $i$ 个组分的摩尔分数, $C_{p, i}$ 为第 $i$ 个组分 的定压比热容. $M_{i}$ 为第 $i$ 个组分的分子量.

\section{2 计算结果分析}

\section{1 污染组分最终产物 $\mathrm{H}_{2} \mathrm{O}$ 的作用}

污染组分最终产物 $\mathrm{H}_{2} \mathrm{O}$ 的工作状况设置见表 2, 结果分析见图 5、图 6. 随着进口空气中的水含量 的增多, 超燃室的燃烧效率、比推力均明显下降.

从化学反应动力学模型可以看出: $\mathrm{H}_{2} \mathrm{O}$ 在链引 发阶段通过反应 $\mathrm{CH}_{3}+\mathrm{H}_{2} \mathrm{O} \rightarrow \mathrm{CH}_{4}+\mathrm{OH}$ 发挥重要作 用; 在链分支阶段, $\mathrm{H}_{2} \mathrm{O}$ 在反应 $\mathrm{HCO}+\mathrm{H}_{2} \mathrm{O} \rightarrow \mathrm{CH}_{2} \mathrm{O}+$

\section{表 2 含 $\mathrm{H}_{2} \mathrm{O}$ 污染空气组分}

Table 2 Vitiation cases containing $\mathrm{H}_{2} \mathrm{O}$

\begin{tabular}{lcccc}
\hline & $x\left(\mathrm{H}_{2} \mathrm{O}\right)$ & $x\left(\mathrm{O}_{2}\right)$ & $x\left(\mathrm{~N}_{2}\right)$ & $\cdots$ \\
\hline Case $1^{\#}$ & 0.011 & 0.21 & 0.779 & 0 \\
Case 2 & 0.050 & 0.21 & 0.740 & 0 \\
Case 3 & 0.112 & 0.21 & 0.678 & 0 \\
Case 4 & 0.224 & 0.21 & 0.566 & 0 \\
\hline
\end{tabular}

" represents bench case (electric heating case); " represents ethanol combustion heating case when the content of each species equals to that at the outlet of heater.

表 3 含 $\mathrm{CO}_{2}$ 污染空气组分

Table 3 Vitiation cases containing $\mathrm{CO}_{2}$

\begin{tabular}{cccccc}
\hline & $x\left(\mathrm{CO}_{2}\right)$ & $x\left(\mathrm{H}_{2} \mathrm{O}\right)$ & $x\left(\mathrm{O}_{2}\right)$ & $x\left(\mathrm{~N}_{2}\right)$ & $\cdots$ \\
\hline Case $1^{\#}$ & 0 & 0.011 & 0.21 & 0.7790 & 0 \\
Case 2 & 0.0712 & 0.011 & 0.21 & 0.7078 & 0 \\
Case 3 & 0.1424 & 0.011 & 0.21 & 0.6366 & 0 \\
Case 4 & 0.2848 & 0.011 & 0.21 & 0.4942 & 0 \\
\hline
\end{tabular}

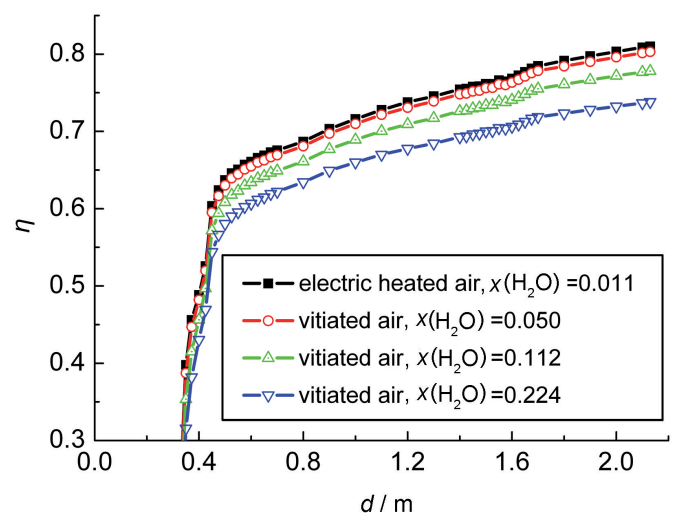

图 $5 \mathrm{H}_{2} \mathrm{O}$ 含量不同时的燃烧效率

Fig.5 Combustion efficiencies at different molar fractions of $\mathrm{H}_{2} \mathrm{O}$

$\mathrm{OH}$ 中也非常关键; 在这两个反应中, $\mathrm{H}_{2} \mathrm{O}$ 的增加促 进了自由基生成, 对燃烧着火过程起到了一定促进 作用, 但作用有限; 在一定的条件下, $\mathrm{H}_{2} \mathrm{O}$ 作为第三 体在反应 $\mathrm{H}+\mathrm{O}_{2}+\mathrm{M} \rightarrow \mathrm{HO}_{2}+\mathrm{M}$ 和 $\mathrm{H}+\mathrm{H}+\mathrm{M} \rightarrow \mathrm{H}_{2}+\mathrm{M}$ 中 碰撞频率因子较高, 造成自由基复合, 因此对燃烧起 负面作用. 另外, 由图 7 可见, 当水含量增加, 平均比 热容会增加, 各截面的质量平均温度下降, 也是导致 燃烧效率下降的一个重要原因. 当燃烧效率下降, 会 使燃气做功能力下降, 从而使燃烧室比推力下降.

根据热力学分析, 由图 7 可见, 平均分子量随 $\mathrm{H}_{2} \mathrm{O}$ 含量的增加而减小, 一定温度条件下, 燃气平均 分子量减小应该促使燃气做功能力增强, 也就是说 从热力学角度分析进口空气中 $\mathrm{H}_{2} \mathrm{O}$ 含量增加会使比 推力增大. 但由于分子量的增加有限, 该影响较小.

综合化学动力学和热力学分析, 定性地说明 $\mathrm{H}_{2} \mathrm{O}$ 对甲烷超燃过程的影响在化学能转化为热能阶

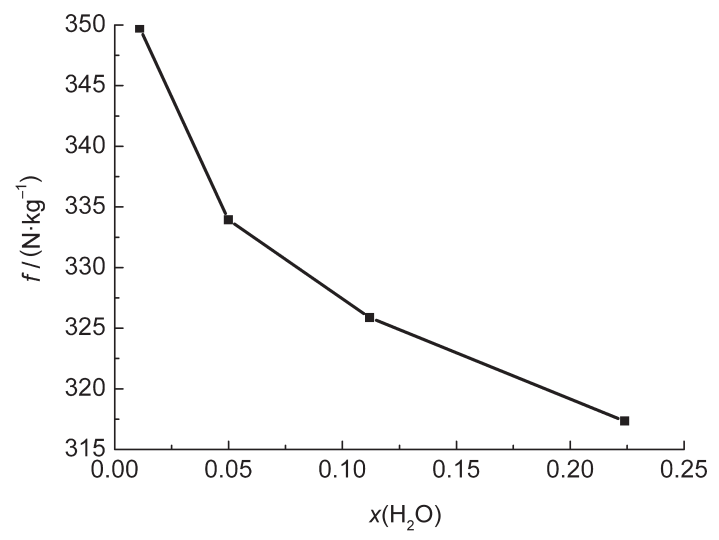

图 $6 \mathrm{H}_{2} \mathrm{O}$ 含量不同时的燃烧室比推力

Fig.6 Combustor specific thrusts at different molar fractions of $\mathrm{H}_{2} \mathrm{O}$

$f$ is the specific thrust, that is defined as the ratio of the combustor thrust to the mass flow-rate. 


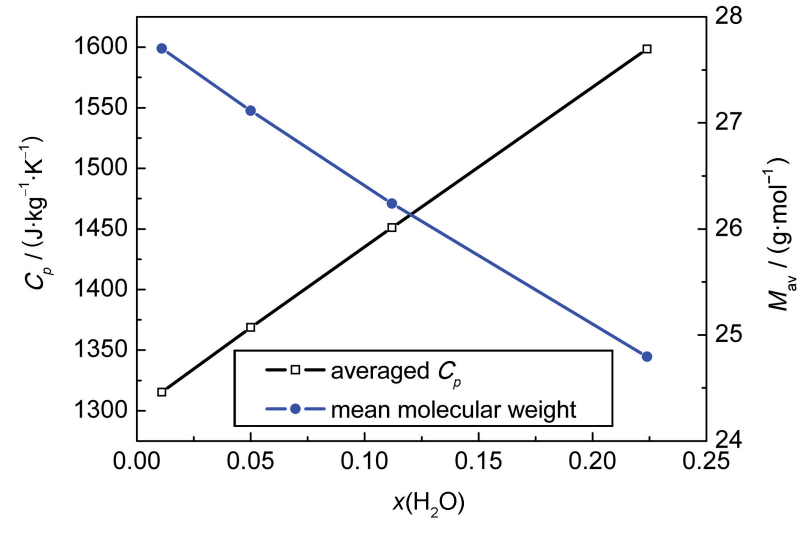

图 $7 \mathrm{H}_{2} \mathrm{O}$ 含量不同时的平均比热容和平均分子量

Fig.7 Averaged specific heat capacities and molecular weight at different molar fractions of $\mathrm{H}_{2} \mathrm{O}$

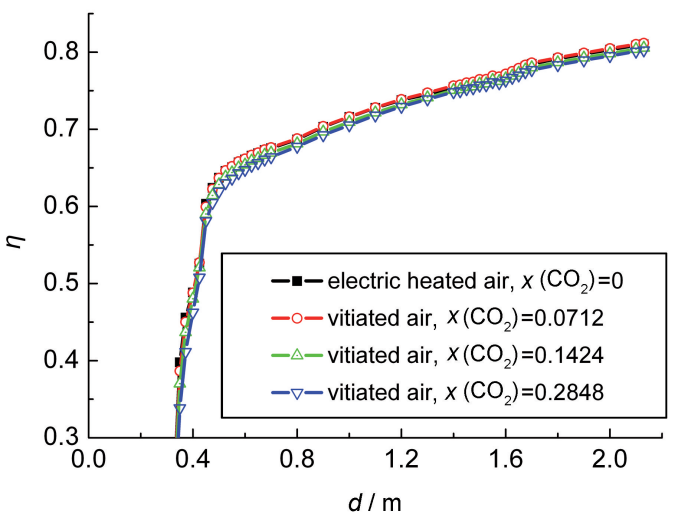

图 $8 \mathrm{CO}_{2}$ 含量不同时的燃烧效率

Fig.8 Combustion efficiencies at different molar fractions of $\mathrm{CO}_{2}$

段起的消极作用强于在热能转化为动能阶段起的积 极作用, 从而降低了甲烷超燃性能.

\section{2 污染组分最终组分 $\mathrm{CO}_{2}$ 的作用}

污染组分最终产物 $\mathrm{CO}_{2}$ 的工况设置见表 3 , 结果 分析见图 8、图9. 从图 8、图9、图10可以看出, 燃烧 效率未随 $\mathrm{CO}_{2}$ 含量增加而明显变化,而比推力明显 下降了, 同时燃气平均分子量增大了. 从化学反应动 力学角度看, $\mathrm{CO}_{2}$ 属于比较惰性的最终产物, 它对燃 烧过程的影响是有限的. 污染物 $\mathrm{CO}_{2}$ 因其大分子量 特性使燃气平均分子量增大, 从而使燃气做功能力 下降, 这是 $\mathrm{CO}_{2}$ 对超燃室燃烧性能起消极作用的主

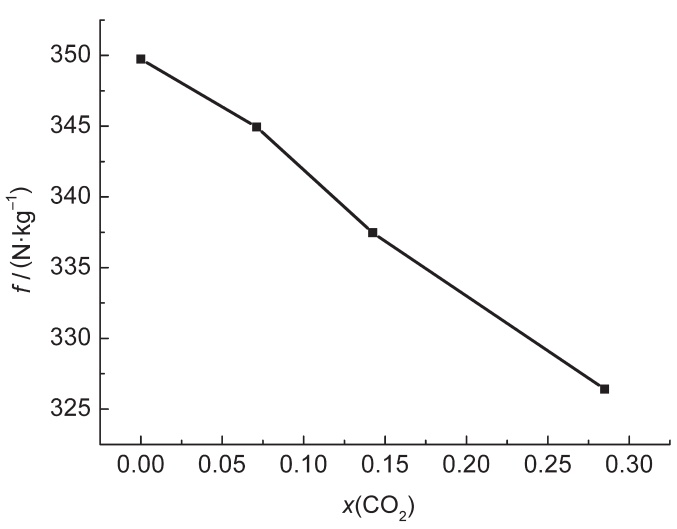

图 $9 \mathrm{CO}_{2}$ 含量不同时的燃烧室比推力

Fig.9 Combustor specific thrusts at different molar fractions of $\mathrm{CO}_{2}$

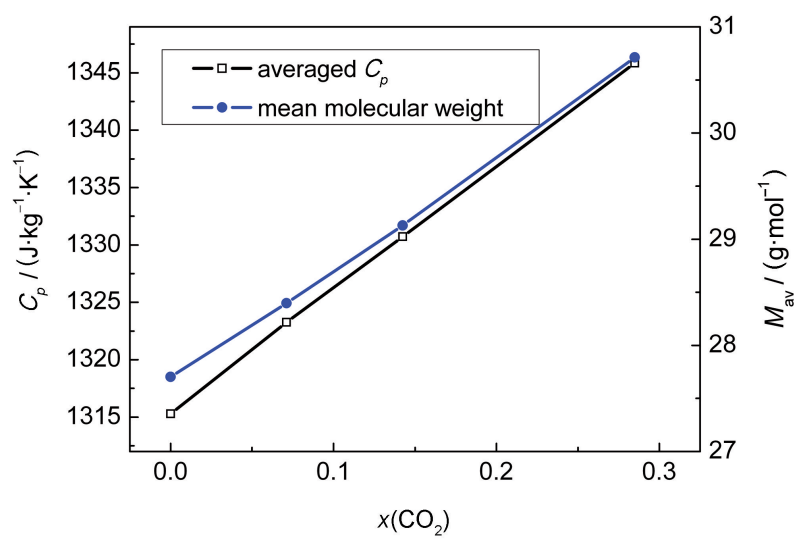

图 $10 \mathrm{CO}_{2}$ 含量不同时的平均比热容和平均分子量

Fig.10 Averaged specific heat capacities and molecular weight at different molar fractions of $\mathrm{CO}_{2}$

要原因。

\section{3 污染组分自由基 $\mathrm{H} 、 O 、 O H$ 的作用}

污染组分自由基 $\mathrm{H} 、 \mathrm{O} 、 \mathrm{OH}$ 的工况设置见表 4 , 结果分析见图 11, 随着进口空气中的自由基 $\mathrm{H} 、 \mathrm{O}$ 和 $\mathrm{OH}$ 含量的增多, 超燃室的燃烧效率均上升.

从化学反应动力学角度, 根据本文化学反应动 力学模型, 说明自由基 $\mathrm{O}$ 主要是通过链分支反应 $\mathrm{CH}_{3}+\mathrm{O} \rightleftharpoons \mathrm{CH}_{2} \mathrm{O}+\mathrm{H}$ 和链传递反应 $\mathrm{O}+\mathrm{H}_{2} \mathrm{O} \rightleftharpoons \mathrm{OH}+\mathrm{OH}$ 来促进燃烧, 从而提升超燃室性能; 自由基 $\mathrm{OH}$ 是非 常活跃的组分, 它主要通过促进链引发反应 $\mathrm{CH}_{4}+$ $\mathrm{OH} \rightleftharpoons \mathrm{CH}_{3}+\mathrm{H}_{2} \mathrm{O}$ 和链分支反应 $\mathrm{CH}_{2} \mathrm{O}+\mathrm{OH} \rightleftharpoons \mathrm{HCO}+$

表4 分别含自由基 $\mathrm{O} 、 \mathrm{OH}$ 和 $\mathrm{H}$ 以及中间产物 $\mathrm{CO}$ 和 $\mathrm{H}_{2}$ 污染空气组分

Table 4 Vitiation cases containing free radicals $\mathrm{O}, \mathrm{OH}$ and $\mathrm{H}$ as well as intermediate products $\mathrm{CO}$ and $\mathrm{H}_{2}$

\begin{tabular}{ccccccccc}
\hline & $x(\mathrm{O})$ & $x(\mathrm{OH})$ & $x(\mathrm{H})$ & $x(\mathrm{CO})$ & $x\left(\mathrm{H}_{2}\right)$ & $x\left(\mathrm{H}_{2} \mathrm{O}\right)$ & $x\left(\mathrm{O}_{2}\right)$ & $x\left(\mathrm{~N}_{2}\right)$ \\
\hline Case $1^{\#}$ & 0 & 0 & 0 & 0 & 0 & 0.011 & 0.21 & $1-\sum x_{i}$ \\
Case 2 & 0.0030 & 0.0031 & 0.0001 & 0.0025 & $0.0002^{*}$ & 0.011 & 0.21 & $1-\sum x_{i}$ \\
Case 3 & $0.0073^{*}$ & $0.0061^{*}$ & $0.0002^{*}$ & $0.0050^{*}$ & 0.0030 & 0.011 & 0.21 & $1-\sum x_{i}$ \\
Case 4 & 0.0145 & 0.0122 & 0.0004 & 0.0300 & 0.0080 & 0.011 & 0.21 & $1-\sum x_{i}$ \\
\hline
\end{tabular}



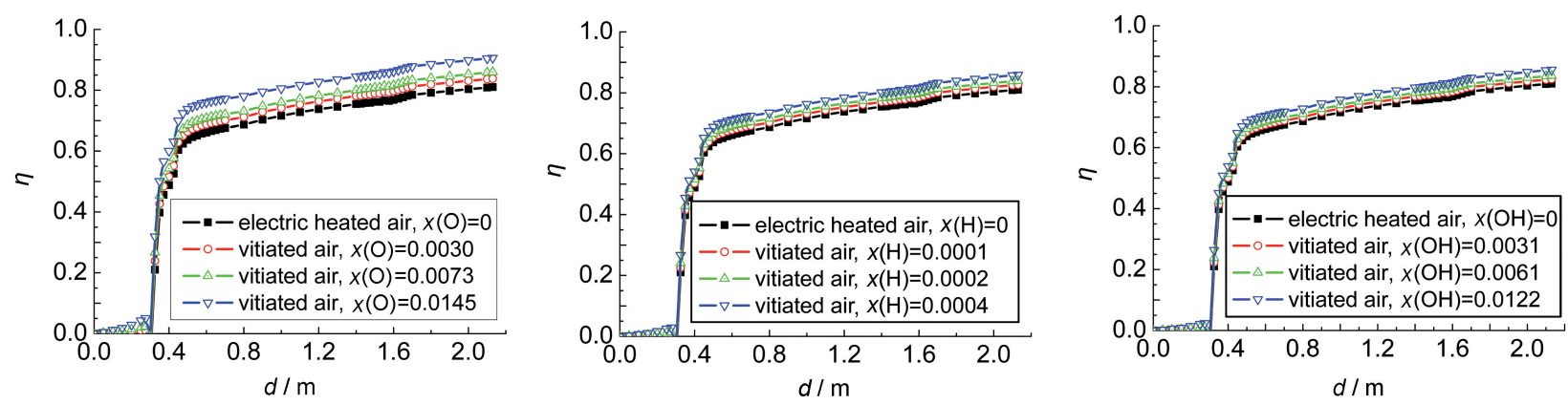

图 11 自由基 $\mathrm{O} 、 \mathrm{H}$ 和 $\mathrm{OH}$ 含量不同时的燃烧效率

Fig.11 Combustion efficiencies at different molar fractions of free radicals $\mathrm{O}, \mathrm{H}$, and $\mathrm{OH}$

$\mathrm{H}_{2} \mathrm{O} 、 \mathrm{CO}+\mathrm{OH} \rightleftharpoons \mathrm{CO}_{2}+\mathrm{H}$ 来促进燃料燃烧过程, 从而 使燃烧效率、比推力上升; 自由基 $\mathrm{H}$ 主要通过链终止 反应 $\mathrm{H}+\mathrm{OH}+\mathrm{M} \rightleftharpoons \mathrm{H}_{2} \mathrm{O}+\mathrm{M}$ 和 $\mathrm{CH}_{3}+\mathrm{H}+\mathrm{M} \rightleftharpoons \mathrm{CH}_{4}+\mathrm{M}$ 发 生作用, 它与甲烷燃烧过程形成了竞争, 抑制了甲烷 的燃烧, 但由于自由基 $\mathrm{H}$ 本身作为相当活跃的燃料 燃烧而使超燃室性能提升.

自由基 $\mathrm{O} 、 \mathrm{OH} 、 \mathrm{H}$ 促进超声速燃烧的能力是不 一样的. $\mathrm{O}$ 的摩尔分数从 0 到 0.0073 , 使燃烧效率提 升 $5.9 \% ; \mathrm{OH}$ 的摩尔分数从 0 到 0.0122 , 使燃烧效率 提升 $5.6 \% ; \mathrm{H}$ 的摩尔分数从 0 到 0.0004 , 使燃烧效率
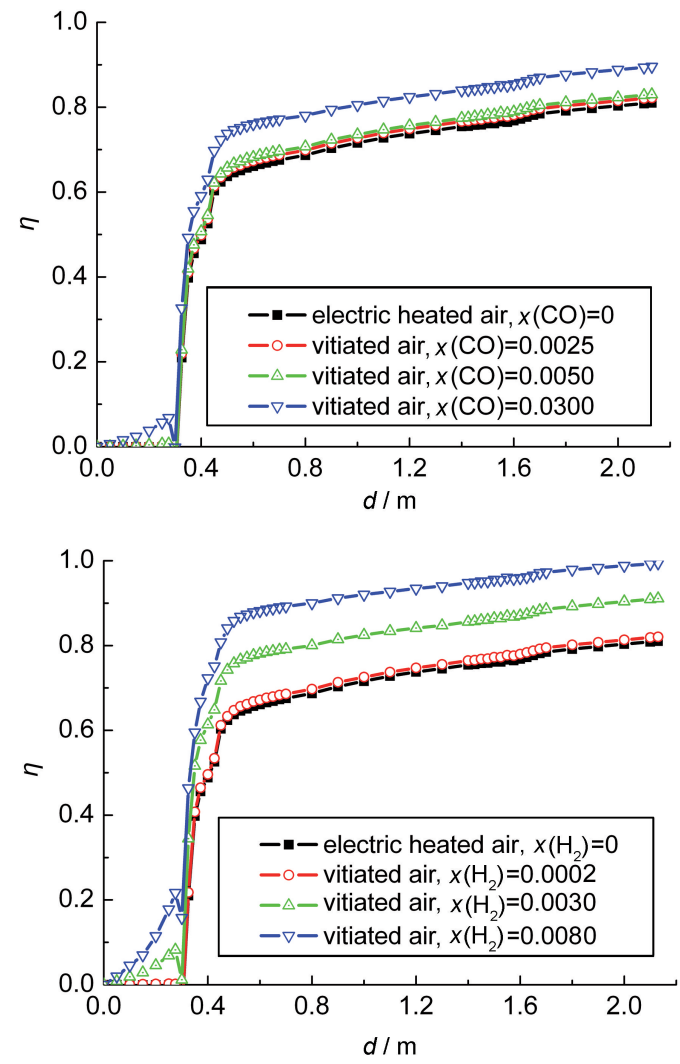

图 12 中间产物 $\mathrm{CO}$ 和 $\mathrm{H}_{2}$ 含量不同时的燃烧效率

Fig.12 Combustion efficiencies at different molar fractions of intermediate products $\mathrm{CO}$ and $\mathrm{H}_{2}$
提升 $6.1 \%$. 于是可以定性地看出, 这三种自由基对 甲烷超声速燃烧效率提升能力强弱应该依次为 $\mathrm{H}$ 、 $\mathrm{O} 、 \mathrm{OH}$.

从热力学角度分析, 由于进口污染空气中自由 基 $\mathrm{O} 、 \mathrm{OH} 、 \mathrm{H}$ 含量非常少, 数量级在 $10^{-3}$ 左右. 因此自 由基 $\mathrm{O} 、 \mathrm{OH} 、 \mathrm{H}$ 含量引起燃气的平均定压比热容和 平均分子量的改变对超燃室做功性能的影响是非常 有限的, 自由基主要通过促进化学反应动力学过程 来提升超燃室的性能.

\section{4 污染组分中间产物 $\mathrm{CO}$ 和 $\mathrm{H}_{2}$ 的作用}

污染组分中间产物 $\mathrm{CO}$ 和 $\mathrm{H}_{2}$ 的工况设置见表 4 , 结果分析见图 12, 随着进口空气中的中间产物 $\mathrm{CO}$ 和 $\mathrm{H}_{2}$ 含量的增多, 超燃室的燃烧效率、比推力均明 显上升了.

因为 $\mathrm{CO}$ 本身是性质比较活跃的燃料, 通过 $\mathrm{OH}+\mathrm{CO} \rightleftharpoons \mathrm{H}+\mathrm{CO}_{2}$ 作为额外添加的燃料提升了甲烷 超燃性能; $\mathrm{H}_{2}$ 对超声速燃烧的作用与 $\mathrm{H} 、 \mathrm{CO}$ 对超声 速燃烧的作用是类似的. $\mathrm{H}_{2}$ 的加入会抑制甲烷的消 耗, 但 $\mathrm{H}_{2}$ 本身通过 $\mathrm{O}+\mathrm{H}_{2} \rightleftharpoons \mathrm{H}+\mathrm{OH}$ 和 $\mathrm{OH}+\mathrm{H}_{2} \rightleftharpoons \mathrm{H}+$ $\mathrm{H}_{2} \mathrm{O}$, 作为添加燃料使超燃室的性能得到提升.

\section{3 结 论}

根据乙醇燃烧加热器燃烧化学反应得到的主要 七种组分, 采用甲烷 18 组分 24 步反应简化机理, 得 到污染空气中最终组分 $\mathrm{H}_{2} \mathrm{O} 、 \mathrm{CO}_{2}$, 自由基 $\mathrm{H} 、 \mathrm{O} 、 \mathrm{OH}$ 和中间产物 $\mathrm{CO} 、 \mathrm{H}_{2}$ 对甲烷超声速燃烧性能的主要 影响如下:

(1) 进口空气中污染组分 $\mathrm{H}_{2} \mathrm{O}$ 含量增多使燃烧 效率、比推力均下降. $\mathrm{H}_{2} \mathrm{O}$ 的增加造成平均比热容增 加, 总温度降低, 并作为第三体抑制甲烷的燃烧过 程, 使超燃室的性能下降.

(2) 进口空气中污染组分 $\mathrm{CO}_{2}$ 含量增多对燃烧 效率没有明显影响, 但使超燃室参考比推力下降. 
$\mathrm{CO}_{2}$ 主要因为其大分子量特性使燃气平均分子量增 大, 从而降低超燃室做功能力, 对甲烷超燃性能起 消极作用.

(3) 进口空气中自由基 $\mathrm{O} 、 \mathrm{OH} 、 \mathrm{H}$ 含量增多均使 超燃室燃烧效率上升. 自由基 $\mathrm{O} 、 \mathrm{OH}$ 主要通过化学 反应动力学过程, 加快燃烧过程, 促进甲烷和煤油 消耗而提高燃烧效率, 从而提升超燃室性能. 自由 基 $\mathrm{H}$ 作为额外添加的活跃燃料对甲烷超燃性能起 积极作用.

(4) 进口空气中污染组分 $\mathrm{CO}$ 和 $\mathrm{H}_{2}$ 均对甲烷超 燃性能起积极作用. 它们的作用原理与自由基 $\mathrm{H}$ 的 作用类似.

\section{References}

1 Edelman, R. B.; Spadaccini, L. J. J. Spacecraft, 1969, 6(12): 1442

2 Mattick, S. J.; Frankel, S. H. Numerical modeling of supersonic combustion_validation and vitiation studies using FLUENT// 41st AIAA/ASME/SAE/ASEE Joint Propulsion Conference \& Exhibit. Tucson, Arizona, 2005: 10-13

3 Tomioka, S.; Hiraiwa, T.; Kobayashi, K.; Izumikawa, M.; Kishida T.; Yamasaki H. J. Propulsion Power, 2007, 23(4): 789

4 Li, W. Q.; Song, W. Y.Journal of Air Force Engineering University,
2006, 5(7): 10 [李卫强, 宋文艳. 空军工程大学学报, 2006, 5(7): $10]$

5 Liu, W. X.; He, W.; Li, H. B.; Li, X. Y.; Le, J. L. Chinese Science Bulletin, 2009, 54(8): 1317 [刘伟雄, 贺 伟, 李宏斌, 李象远, 乐嘉陵. 科学通报, 2008, 53(8): 2257]

6 Shao, J. X.; Tan, N. X.; Liu, W. X.; Li, X. Y. Acta Phys. -Chim. Sin., 2010, 26(2): 270 [邵菊香, 谈宁馨, 刘伟雄, 李象远. 物理 化学学报, 2010, 26(2): 270]

7 Liu, O. Z.; Cai, Y. H.; Hu, Y. L.; Liu, J. H.; Ling, W. H. Journal of Propulsion Technology, 2004, 25(5): 463 [刘欧子, 蔡元虎, 胡欲 立, 刘敬华, 凌文辉. 推进技术, 2004, 25(5): 463]

8 Peters, N.; Kee, R. J. Combust. Flame, 1987, 68: 17

9 Tong, G.; Huang, Y.; Chen, Y. L. Journal of Fuel Chemistry and Technology, 2000, 28(1): 49 [董 刚, 黄 鹰, 陈义良. 燃料化 学学报, 2000, 28(1): 49]

10 Bowman, C. T.; Hanson, R. K.; Davidson, D. F.; Gardiner Jr., W. C.; Lissianski, V.; Smith, G. P.; Golden, D. M.; Frenklach, M.; Goldenberg, M. 1994, http://www.me.berkeley.edu/gri_mech/

11 Marinov, N. M. Int. J. Chem. Kinet., 1999, 31(3):183

12 Davidenko, D. M.; Gökalp, I.; Dufour, E.; Magre, P. Systematic numerical study of the supersonic combustion in an experimental combustion chamber. 14th AIAA/AHI Space Planes and Hypersonic Systems and Technologies Conference, AIAA 2006-7913 